\title{
El nutritionDay en Latinoamérica
}

\author{
The nutritionDay in Latin America \\ O nutritionDay na América Latina
}

\begin{abstract}
Angélica María Pérez Cano', Evelyn Frias-Toral ${ }^{2}$, María Cristina Gonzalez ${ }^{3}$, Silvana Paiva Orlandi ${ }^{4}$, Claudia P. Maza Moscoso5, Gabriela Cucalón 6 .
\end{abstract}

https://doi.org/10.35454/rncm.v4n4.357

\section{¿QUÉ ES EL nutritionDay?}

El nutritionDay es una iniciativa de la Sociedad Europea de Nutrición Clínica y Metabolismo (ESPEN) y la Universidad de Viena, que desde 2006 pretende luchar contra la desnutrición hospitalaria ${ }^{(1)}$. A través de la recolección de información en un día, busca también mejorar el cuidado nutricional de los pacientes hospitalizados y generar conciencia sobre la importancia de la desnutrición asociada con la enfermedad. El nutritionDay es un estudio de tipo auditoría, multicéntrico e internacional que permite comparar las instituciones de salud consigo mismas y con instituciones en el mundo de una manera anónima y sin costo alguno. Participar cada año en este estudio es importante para poder observar la evolución y compararse con la referencia internacional y, de este modo, crear estrategias para mejorar los procesos de cuidado nutricional $^{(1)}$. En la Tabla 1 se muestran las características del nutritionDay.

Coordinadora, nutritionDay Colombia, Jefe del departamento de nutrición, Hospital Universitario Infantil de San José, presidente de la Asociación Colombiana de Nutrición Clínica. Bogotá, Colombia.

2 Coordinadora, nutritionDay Ecuador. Escuela de Medicina, Universidad Católica Santiago de Guayaquil, Ecuador.

3 Coordinadora, nutritionDay Brasil. Profesora titular, Universidad Católica de Pelotas, Brasil.

4 Departamento de Nutrición, Universidad Federal de Pelotas, Brasil.

5 Coordinadora nutritionDay, Guatemala. Jefe de la sede de nutrición clínica, Centro Médico Militar, Guatemala. Profesora de Nutrición Clínica, Universidad del Valle de Guatemala.

6 Escuela Superior Politécnica del Litoral (ESPOL), Facultad de Ciencias de la Vida. Guayaquil, Ecuador.
Tabla 1. Características del nutritionDay

\begin{tabular}{|l|}
\hline El nutritionDay es: \\
\hline $\begin{array}{l}\text { - una iniciativa mundial para luchar contra la desnutrición en } \\
\text { las instituciones de salud }\end{array}$ \\
\hline $\begin{array}{l}\text { - un programa científico: la base de datos está disponible } \\
\text { para los investigadores, científicos y educadores }\end{array}$ \\
\hline $\begin{array}{l}\text { - una auditoria transversal de un día con evaluación de los } \\
\text { desenlaces }\end{array}$ \\
\hline $\begin{array}{l}\text { - un ciclo continuo de mejora: registrarse en nutritionDay, } \\
\text { recolectar la información, obtener el reporte y beneficiarse }\end{array}$ \\
\hline $\begin{array}{l}\text { - cuatro opciones: nutritionDay en los servicios de hospitales, } \\
\text { servicios oncológicos, UCl y hogares geriátricos }\end{array}$
\end{tabular}

UCl: unidades de cuidados intensivos.

El nutritionDay ha permitido conocer que hasta el $40 \%$ de los pacientes hospitalizados se ve afectado por desnutrición relacionada con la enfermedad, lo cual puede conducir a un aumento de los costos de la atención médica por causas de salud, una estancia prolongada y un pronóstico desfavorable ${ }^{(1)}$. Es común que la desnutrición esté presente en personas que se vean físicamente delgadas y con poco acceso a alimentos de buena calidad e ingesta insuficiente. Sin embargo, incluso las personas con exceso de peso pueden tener una ingesta nutricional insuficiente que, al estar acompañado de una patología aguda o crónica, aumente el riesgo de complicaciones durante su hospitalización, tal como puso en evidencia la pandemia actual, en la que la mortalidad fue mayor en pacientes con obesidad y enfermedad por coronavirus de 2019 (COVID-19); una situación similar se observa 
en pacientes con cáncer de ovario y mamas ${ }^{(2,3)}$. En estos casos, el peso del paciente y su curso de tratamiento nutricional puede malinterpretarse y tanto el riesgo como la desnutrición pueden no detectarse. Es por esto que deben implementarse procesos de cuidado nutricional que permitan un manejo adecuado de la desnutrición asociada con la enfermedad.

\section{¿QUIÉN PUEDE PARTICIPAR?}

Todos los servicios hospitalarios, los servicios de oncología, las UCI y las residencias geriátricas pueden participar. Se incluyen los pacientes que estén hospitalizados el día del nutritionDay.

En Latinoamérica, varios países, entre ellos Colombia, Ecuador y Brasil, tienen una trayectoria importante participando en el nutritionDay desde hace varios años. A estos países se han agregado otros, como el caso de Guatemala, que se unió en 2019. El 2020 fue un año diferente debido a la pandemia de la COVID-19. Sin embargo, esto no impidió que en Latinoamérica se unieran varias instituciones a esta iniciativa global. Todo esto con el propósito de crear conciencia sobre la malnutrición asociada con la enfermedad en nuestros países y, de este modo, poder mejorar la calidad de atención de nuestros pacientes hospitalizados.

Colombia participa en esta iniciativa desde 2009 y ha podido obtener resultados de más de 14000 pacientes, en promedio de 50 instituciones, liderado actualmente por la Licenciada Angélica Pérez de la Asociación Colombiana de Nutrición Clínica (ACNC). Cada año la ACNC ayuda a las instituciones participantes en la creación de matrices de riesgo nutricional que plantee actividades de mejora desde la calidad en la atención nutricional, lo cual ha logrado impactos positivos para las instituciones y para el proceso de cuidado nutricional que reflejan los resultados del nutritionDay. Desde 2015 han logrado incluir, a nivel global, la segunda muestra de pacientes más importante, con un promedio de 2000 a 2300 pacientes anualmente, $y$ aun en pandemia pudieron mantener la participación con 801 pacientes. Colombia ha realizado publicaciones importantes que han permitido tener un panorama del riesgo de la desnutrición hospitalaria en el país y el impacto en los desenlaces ${ }^{(4,5)}$. En las Figuras 1 y 2 se muestran colaboradores de algunos centros de nutritionDay Colombia.

A partir de estos resultados, la ACNC pretende llegar a las entidades gubernamentales y desarrollar políticas y programas que busquen promover una cultura institucional que valore el cuidado nutricional. De esta forma, se busca que se respete el derecho fundamental al cuidado nutricional $y$, por ende, que todos los pacientes reciban una terapia nutricional óptima y oportuna. Desde hace varios años se ha podido apoyar a otros países en la realización del nutritionDay, con lo cual Latinoamérica ha podido beneficiarse de la experiencia de Colombia.

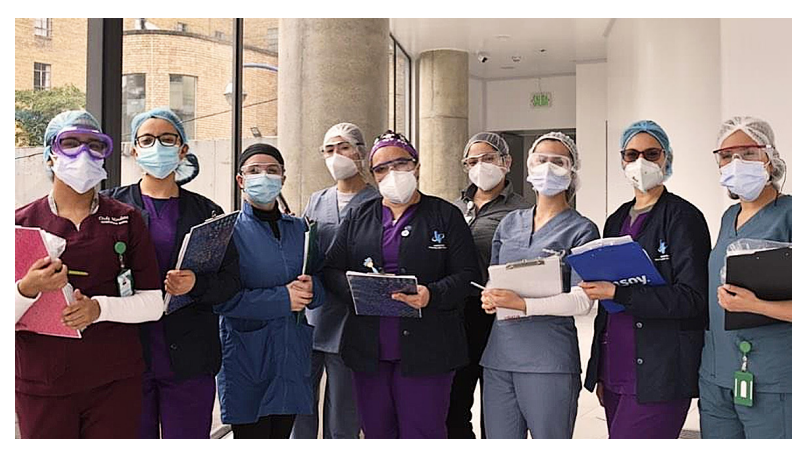

Figura 1. nutritionDay en Colombia, Hospital San Carlos, Bogotá. Foto de archivo de nutritionDay.

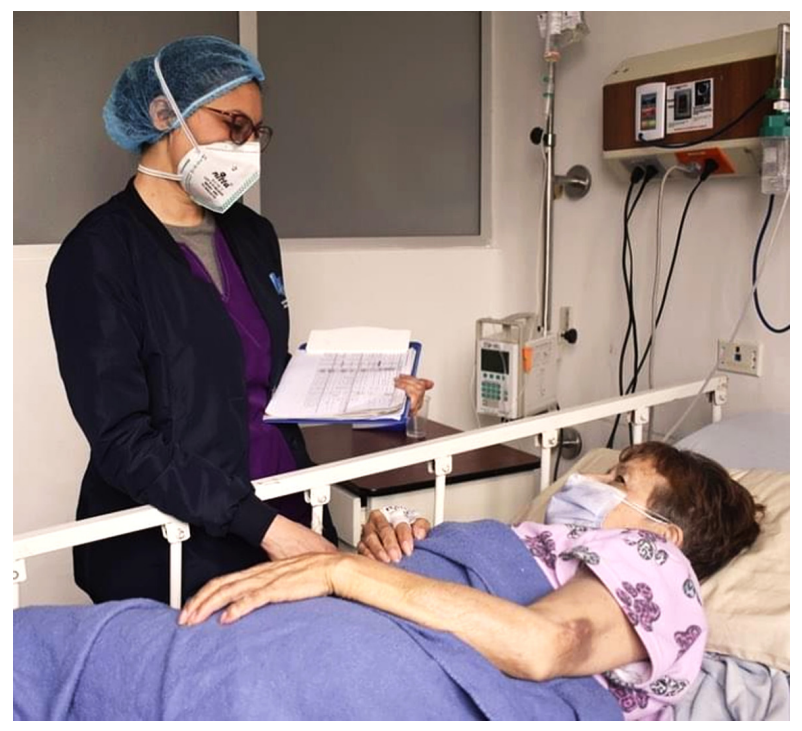

Figura 2. nutritionDay en Colombia, Hospital San Carlos, Bogotá. Foto de archivo de nutritionDay.

En Ecuador se realizó por primera vez el nutritionDay en el Hospital Sociedad de Lucha Contra el Cáncer (SOLCA) en febrero de 2017. A partir de esa fecha, SOLCA participa continuamente en el nutritionDay y, junto con el apoyo de la Asociación Ecuatoriana de Nutrición Parenteral y Enteral (ASENPE), se impulsa la participación de más instituciones de diversas provincias del país. Para dicho efecto se gestionan capacitaciones presenciales y virtuales con varios centros hospitalarios. Es así que hasta la fecha se cuenta con 15 instituciones participantes, entre las cuales están hospitales, uni- 
versidades y asilos de ancianos. Esta colaboración ha permitido que haya un incremento constante en la inclusión de pacientes, logrando incorporar a más de 600 pacientes al último corte realizado a inicios de octubre de 2021, lo que ha beneficiado a los centros participantes al recibir sus reportes completos generados por el nutritionDay para conocer los puntos críticos a mejorar en cada unidad a fin de reducir la malnutrición hospitalaria detectada. Esta tendencia creciente de centros participantes es realmente destacable considerando todas las dificultades que impuso la pandemia desde marzo de 2020. En la Figura 3 se muestran colaboradores de algunos centros del nutritionDay Ecuador.

En Guatemala, desde 2012 existen datos de instituciones que se involucraron en algún momento en el nutritionDay; sin embargo, no ha sido de manera continua. El nutritionDay debe ser una actividad de varias instituciones del país ya que el contexto y carac- terísticas de cada una es diferente. En 2020 se realizó la convocatoria para que las instituciones guatemaltecas se unieran a esta iniciativa, todo esto con el apoyo de la Junta Directiva de la Asociación de Nutricionistas de Guatemala (ANDEGUAT) y de la ACNC. A la convocatoria respondieron 10 instituciones, de las cuales 4 participaron. Se evaluó un total de 173 pacientes de hospitales, 21 pacientes de UCI y 12 de residencias de adulto mayor. Se espera que en 2021 se agreguen más instituciones, ya que ha surgido el interés a raíz de la difusión de la información, capacitaciones nacionales y conferencias sobre el nutritionDay en el país y en países como Colombia y Ecuador, que han extendido la invitación a participar en sus capacitaciones. En las Figuras 4 y 5 se muestran colaboradores de algunos centros de nutritionDay Guatemala.

En Brasil, el nutritionDay empezó en 2009, a partir de una iniciativa coordinada por la Dra. María Cristina
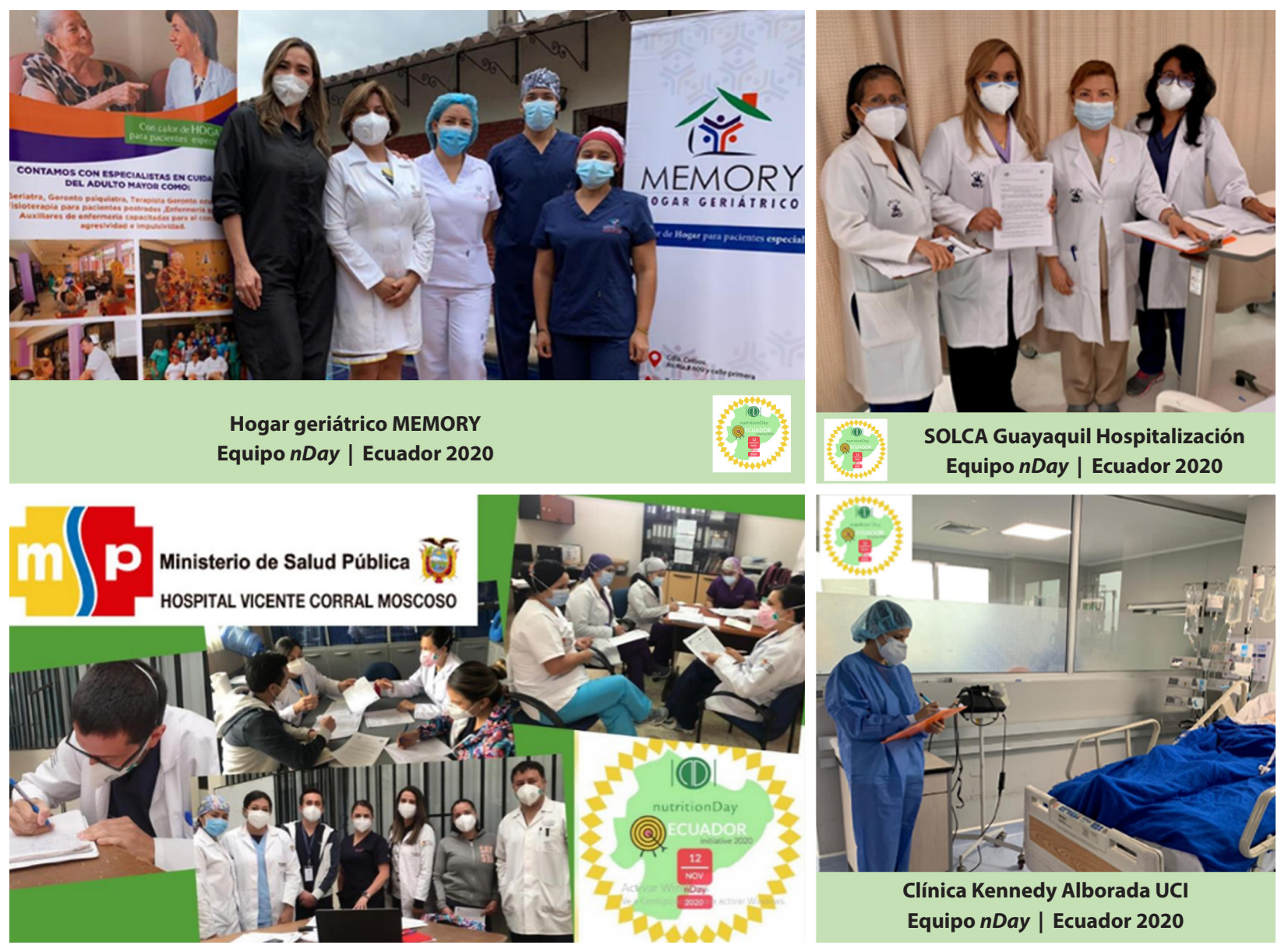

Figura 3. Colaboradores de diferentes centros participantes en el nutritionDay Ecuador. Foto de archivo de nutritionDay. 
González. La iniciativa contó con el apoyo de la Sociedade Brasileira de Nutrição Parenteral e Enteral (BRASPEN) para una amplia difusión de la iniciativa. Durante estos años, se registraron 112 instituciones de salud brasileñas. En las Figuras 6 y 7 se muestran colaboradores de algunos centros del nutritionDay Brasil.

\section{ACTIVIDADES DESTACADAS EN LA REGIÓN}

Colombia ha realizado exitosamente convocatorias para participar en nutritionDay, así como capacitaciones completas para los encuestadores por varios años consecutivos, que han beneficiado no solo a las instituciones a nivel nacional sino también a las de Latinoamérica.

En septiembre de 2020, nutritionDay Ecuador, con el apoyo de ASENPE y la Federación Latinoamericana de

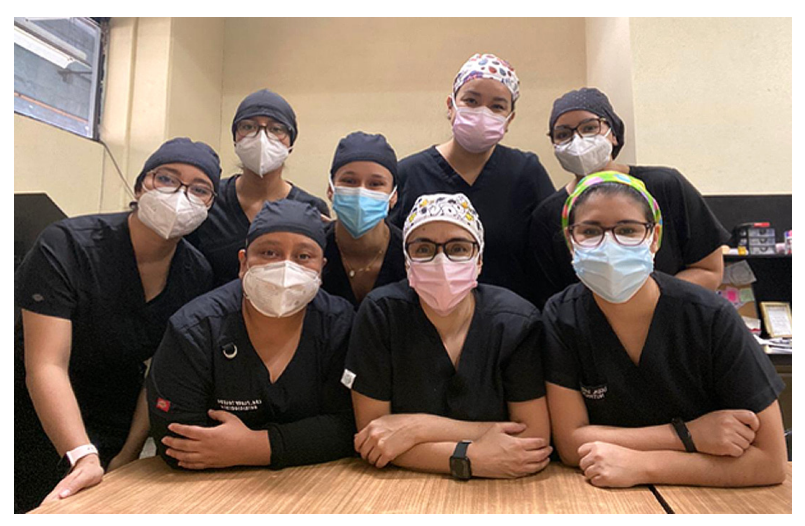

Figura 4. nutritionDay en Guatemala, Hospital General San Juan de Dios, Guatemala. Foto de archivo de nutritionDay.

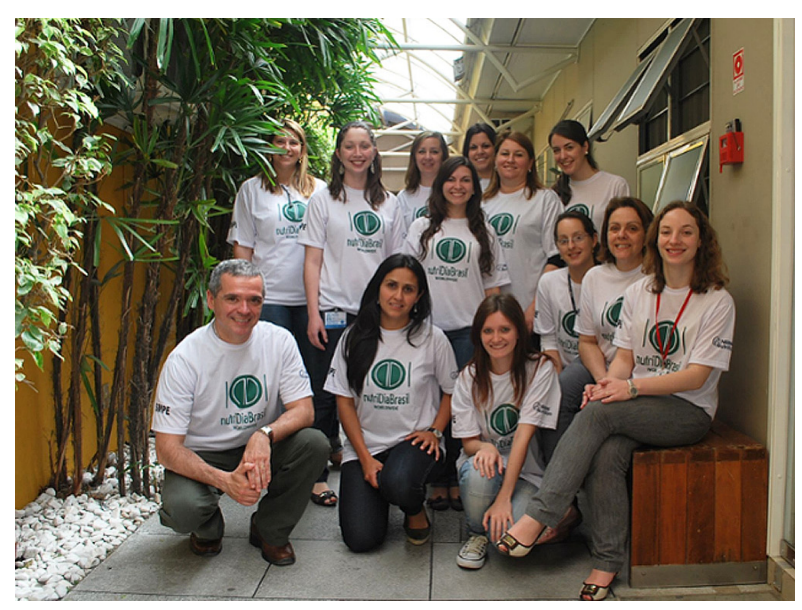

Figura 6. nutritionDay Brasil, Hospital Escola da Universidade Federal de Pelotas/Empresa Brasileña de Servicios Hospitalarios. Foto de archivo de nutritionDay.
Terapia Nutricional, Nutrición Clínica y Metabolismo (FELANPE), organizaron el primer webinar informativo de nutritionDay, el cual contó con la participación de 250 interesados tanto de Ecuador como de varios países latinoamericanos. En este evento se explicaron los procedimientos para cada categoría en la que las instituciones pueden participar en el nutritionDay, se compartieron experiencias de la práctica y se generaron nuevas oportunidades de colaboración, lo que permitió dejar un material para que otras instituciones hispanohablantes pudieran tener los insumos necesarios para capacitarse y participar.

Colombia y Ecuador se han mostrado muy activos generando artículos científicos, pósteres y conferencias socializando los datos del nutritionDay ${ }^{(4-6)}$. Además, a mediados de este año, nutritionDay Ecuador junto con la Escuela Politécnica del Litoral (ESPOL) realizaron la tra-

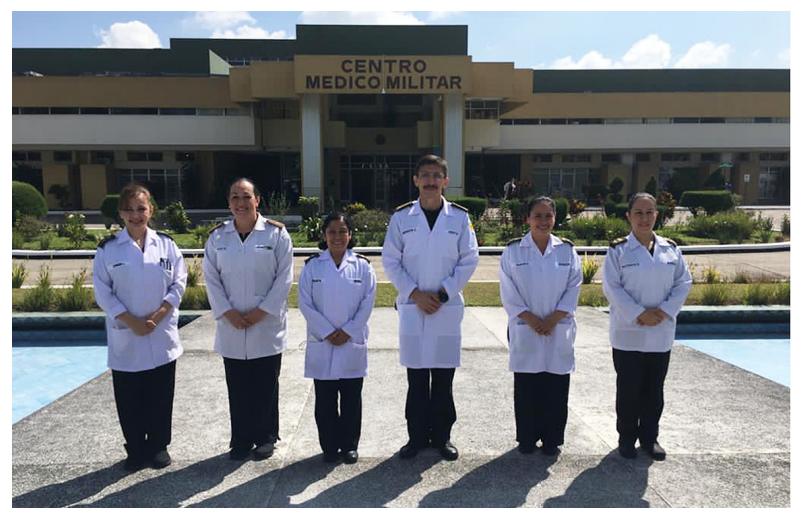

Figura 5. nutritionDay en Guatemala, Hospital Centro Médico Militar. Foto de archivo de nutritionDay.

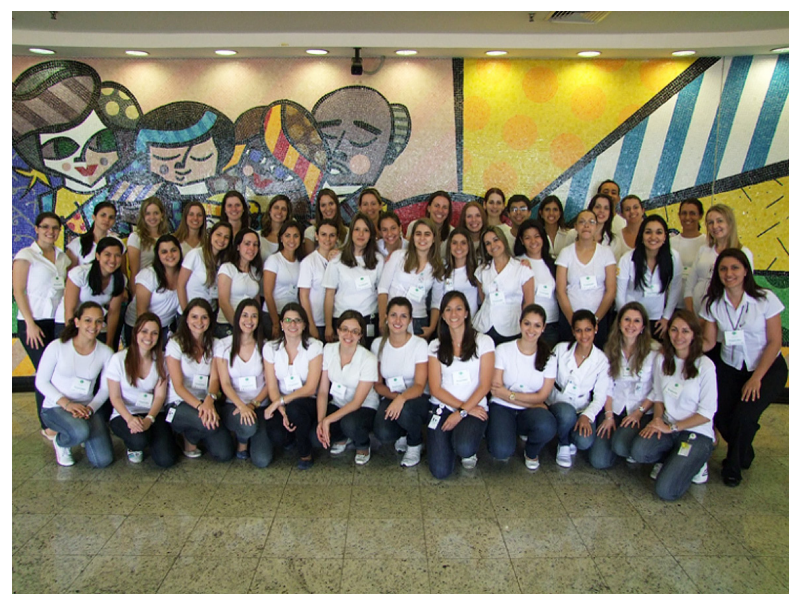

Figura 7. nutritionDay Brasil, Instituto do Cãncer do Estado de São Paulo (ICESP). Foto de archivo de nutritionDay. 
ducción al español de la presentación de entrenamiento para el nutritionDay versión 2021, para poder facilitar dicho material a los países de habla hispana.

\section{NUTRITIONDAY: NUEVAS PROPUESTAS}

El 2021 ha sido un año de aprendizaje y resiliencia para todos, incluidos los que participamos activamente en el nutritionDay. Es por eso que se han realizado muchas alianzas estratégicas para continuar con este gran proyecto. Es así que varios coordinadores nacionales decidimos trabajar en conjunto y realizar actividades que ayuden a que más instituciones de toda Latinoamérica se sumen a esta iniciativa. Para empezar, se planificó un webinar informativo al que asistieron varios países para brindar información general sobre el nutritionDay y las categorías en las cuales pueden participar las diferentes instituciones interesadas. Además, se plantearon las siguientes propuestas a fin de sumar esfuerzos y promover la participación de más organizaciones de la región en el nutritionDay:
- realizar webinars informativos (en español y portugués) mínimo 2 veces por año,

- organizar cursos de capacitación cada 2 meses hasta octubre de cada año (siguiendo el modelo de ACNC),

- manejar redes sociales de forma unificada (la página de Facebook nutritionDay Ecuador pasa a nutritionDay Latinoamérica),

- facilitar la socialización de información de nutritionDay en español/portugués a través de las asociaciones y sociedades de nutrición parenteral y enteral,

- gestionar la publicación de noticias de nutritionDay (RNCM),

- participar activamente en la socialización de resultados de nutritionDay a través de la publicación de artículos, pósteres científicos y conferencias.

En las Figuras 8 y 9 se muestran imágenes del webinar informativo nutritionDay Latinoamérica, realizado el 18 de octubre, el cual contó con más de 200 participantes de Latinoamérica.

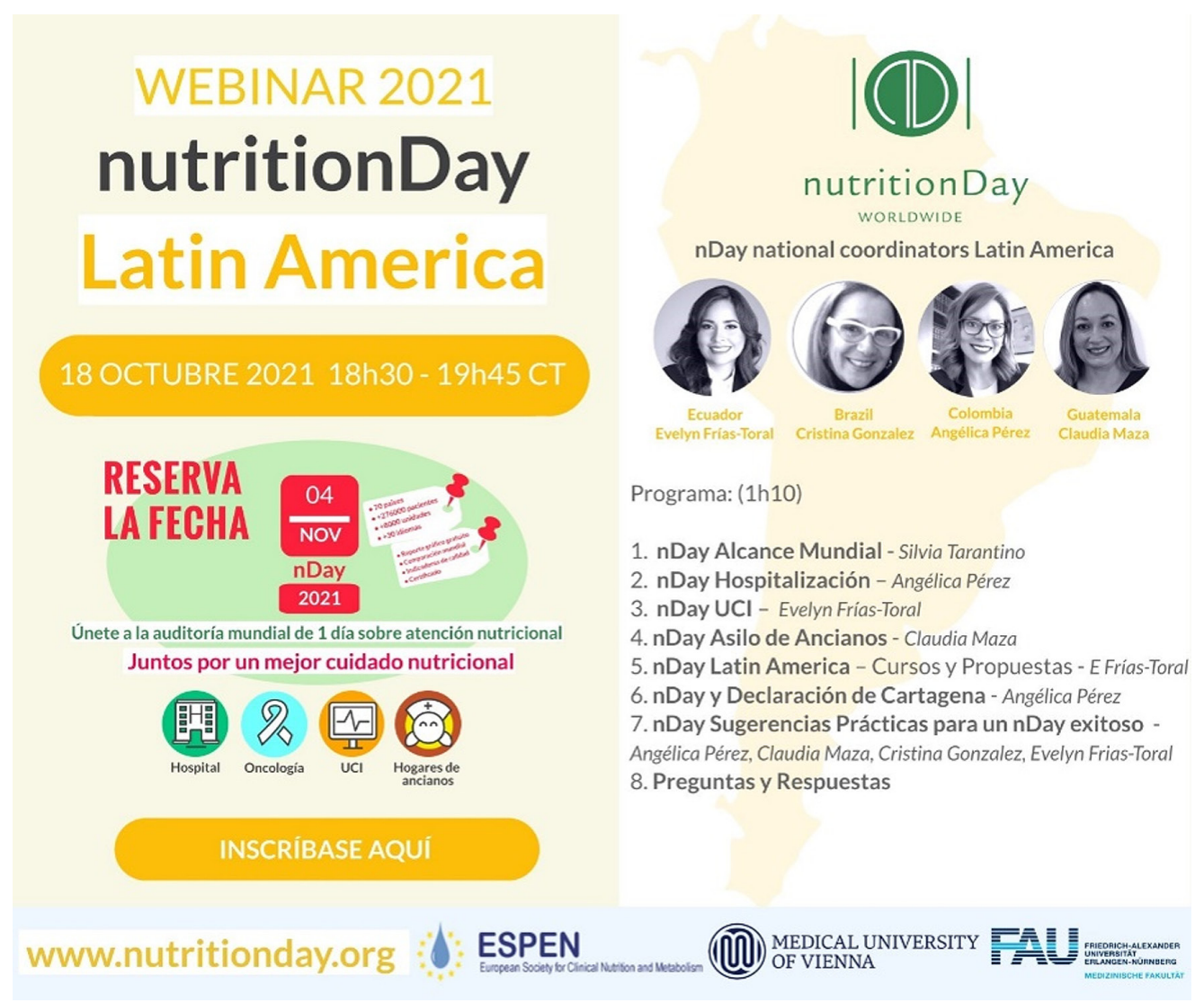

Figura 8. Webinar informativo sobre el nutritionDay para Latinoamérica, realizado el 18 de octubre de 2021. Foto de archivo de nutritionDay. 


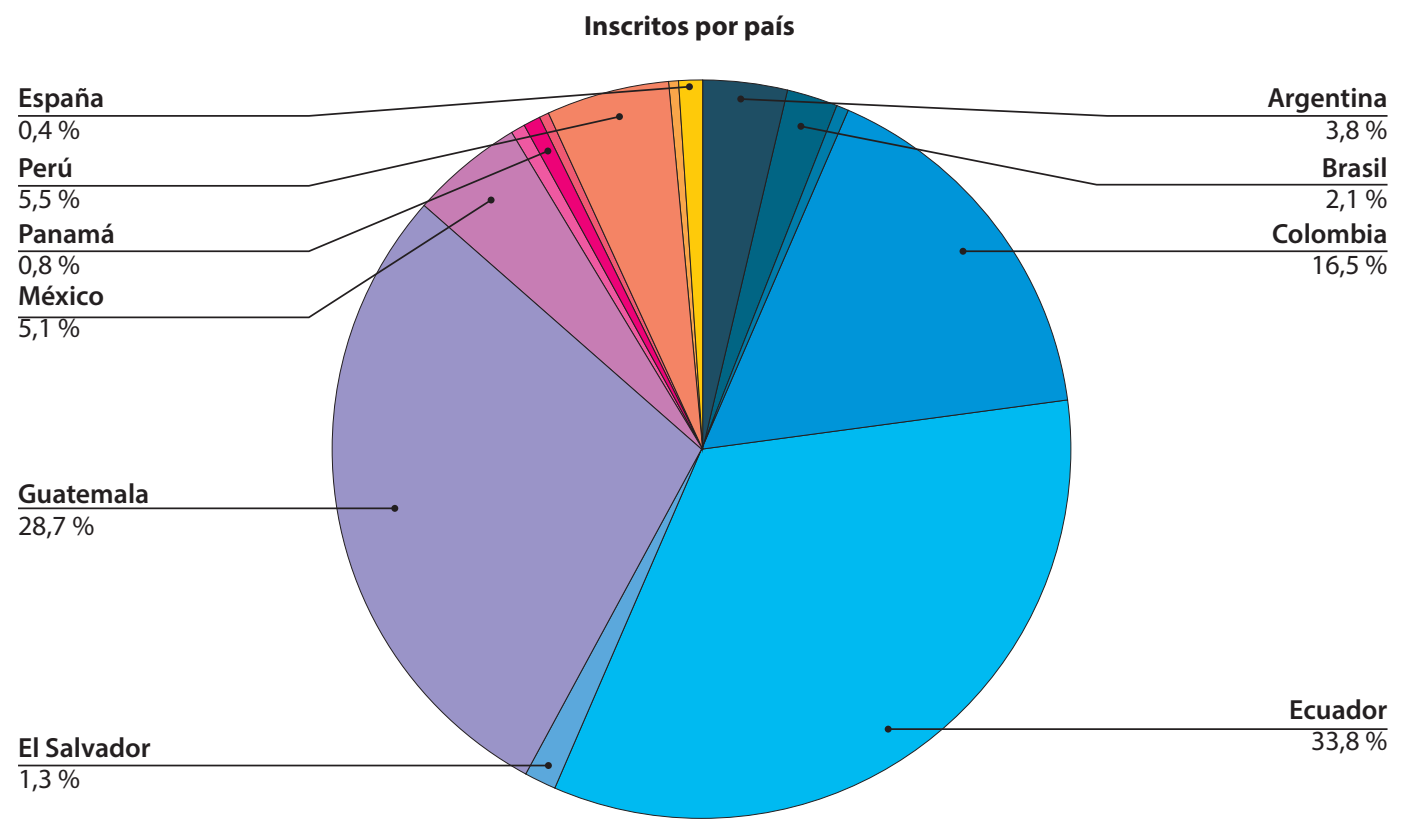

\begin{tabular}{|c|c|c|c|c|c|c|c|c|c|c|c|c|c|c|c|}
\hline Países & Argentina & Brasil & Chile & Colombia & Ecuador & El Salvador & Guatemala & México & Nicaragua & Panamá & Paraguay & Perú & $\begin{array}{c}\text { República } \\
\text { Dominicana }\end{array}$ & España & Total \\
\hline Inscritos & 5 & 2 & 1 & 35 & 70 & 3 & 57 & 8 & 1 & 2 & 1 & 13 & 1 & 1 & 200 \\
\hline
\end{tabular}

Figura 9. Participantes por país en el webinar regional para Latinoamérica. Participantes: 200 personas. Imagen de archivo de nutritionDay.

El nutritionDay nos ha enseñado que, dentro de las instituciones, a pesar de que haya voluntad de hacer las cosas bien, participar en estas actividades evalúa las prácticas y esfuerzos propios de las instituciones, evidenciando muchas oportunidades de mejora. Además, destaca la importancia del diagnóstico de la malnutrición, el cual debe inculcarse desde las aulas de la educación a profesionales de la salud de todo tipo de especialidad.

Estamos conscientes de que con esfuerzos en conjunto podremos lograr más cambios en la atención nutricional de los pacientes. Quienes nos dedicamos a la nutrición clínica tenemos la fuerte convicción de que, en Latinoamérica, se pueden hacer las cosas mucho mejor y que el proceso de cuidado nutricional es un derecho humano ${ }^{(7,8)}$. Participar en este tipo de iniciativas es algo muy positivo porque ayuda a crear conciencia sobre la malnutrición asociada con la enfermedad y a auditar nuestros procesos de atención.

Expresamos nuestro agradecimiento a todas las instituciones participantes del nutritionDay y esperamos que sigan trabajando a favor de la prevención, tamizaje y tratamiento oportuno de la malnutrición. El próximo año esperamos empezar la convocatoria desde los primeros meses para que todas las instituciones interesadas puedan completar todos los trámites requeridos. El trabajo en equipo y la unión de varios países con un mismo fin dará resultados para el beneficio de todos.

\section{¿CÓMO PARTICIPAR?}

Si desea participar, puede contactar a las coordinadoras de cada país para más información.

- Colombia: Licenciada Angélica Pérez Cano. Correo electrónico: nutritionday@nutriclinicacolombia.org

- Ecuador: Dra. Evelyn Frías. Correo electrónico: ndayecuador@gmail.com

- Guatemala: Licenciada Claudia P. Maza Moscoso. Correo electrónico: cmaza34@hotmail.com

- Brasil: Dra. María Cristina González. Correo electrónico: cristinagbs@hotmail.com

\section{Referencias}

1. Hiesmayr M, Tarantino S, Moick S, Laviano A, Sulz I, Mouhieddine $\mathrm{M}$, et al. Hospital Malnutrition, a Call 
for Political Action: A Public Health and NutritionDay Perspective. J Clin Med. 2019;8(12):2048. doi: 10.3390/ jcm8122048

2. Frias-Toral E, Garcia E, Morocho C, Peralta F, Ramirez Y, Fuentes D. Association of the nutric score with 30 and 60-day mortality in sars Covid 19 critically ill patients in an intensive care unit, in Guayaquil - Ecuador. Clin Nutr ESPEN. 2020;40:622. doi: 10.1016/j.clnesp.2020.09.648

3. Rodriguez D, Guerrero M, Maldonado M, Herrera S, Frías E, Santana S. Recomendaciones en el tratamiento nutricional para pacientes oncológicos ante COVID-19.J Health Med Sci. 2020;6(4):303-14.

4. Cardenas D, Bermúdez C, Pérez A, Diaz G, Cortés LY, Contreras CP, et al. Are traditional screening tools adequate for monitoring the nutrition risk of in-hospital patients? An analysis of the nutritionDay database. JPEN J Parenter Enteral Nutr. 2021. doi: 10.1002/jpen.2085

5. Cardenas D, Bermúdez C, Pérez A, Diaz G, Cortes LY, Contreras $\mathrm{CP}$, et al. Nutritional risk is associated with an increase of in-hospital mortality and a reduction of being discharged home: Results of the 2009-2015 nutritionDay survey. Clin Nutr ESPEN. 2020;38:138-45. doi: 10.1016/j. clnesp.2020.05.014

6. Frias-Toral E, Rodriguez D, Guerrero M, Cucalón G, Carvajal D, Jimenez A, et al. The results of nutritionday 2019 in Ecuador. Clin Nutr ESPEN. 2020;40:472-3. doi: 10.1016/j. clnesp.2020.09.200

7. Cárdenas D, Bermúdez CE, Echeverri S, Pérez A, Puentes M, López L, et al. Declaración de Cartagena. Declaración Internacional sobre el Derecho al Cuidado Nutricional y la Lucha contra la Malnutrición [Cartagena Declaration. The International Declaration on the Right to Nutritional Care and the Fight against Malnutrition]. Nutr Hosp. 2019;36(4):974980. doi: 10.20960/nh.02701

8. Cardenas D, Correia MITD, Ochoa JB, Hardy G, RodriguezVentimilla D, Bermúdez CE, et al. Clinical nutrition and human rights. An international position paper. Clin Nutr. 2021;40(6):4029-36. doi: 10.1016/j.clnu.2021.02.039 